\title{
An APG-MUSIC Algorithm Based-on Optimized Sampling Array
}

\author{
Mengxia $\mathrm{Li}^{1}$, Wen $\mathrm{Hu}^{1}$, Jiaying $\mathrm{Di}^{1}$ and Hongtao $\mathrm{Li}^{2}$ \\ ${ }^{1}$ Nanjing University of Aeronautics and Astronautics, Nanjing,Jiangsu,China \\ ${ }^{2}$ Nanjing University of Science and Technology, Nanjing,Jiangsu,China
}

\begin{abstract}
This paper proposes a novel two-dimensional direction of arrival (2D-DOA) estimation with optimized sparse sampling array, which is combined with Accelerated Proximal Gradient singular value thresholding(APG) and Multiple Signal Classification(MUSIC). Firstly, a signal model of 2D-DOA estimation in sparse array is established, which is proved to satisfy low rank feature and NULL Space Property(NSP). Then, Genetic algorithm (GA) is applied to a sparse sampling array to optimize the performance of matrix completion(MC). Finally, MUSIC combined with APG is studied to recover received signal matrix and estimate the direction of arrival. The results of computer simulation demonstrate that compared with conventional 2D-DOA algorithms, the proposed algorithm reduces the number of array elements needed dramatically and effectively lowers the average sidelobes level of spatial spectrum.
\end{abstract}

\section{Introduction}

Direction of arrival(DOA), an important branch of array signal processing $[1,2]$ has received a lot of research achievements and wide applications in electronic reconnaissance, sensor networks, radar signal processing etc. In recent years, DOA estimation has been facing the urgent demand of high precision, high resolution and high robustness. In response to this trend, twodimensional(2D) DOA estimation[3,4] has aroused the interest of the majority of scholars and emerged a large number of estimation methods.

Matrix completion(MC)[5-9], a data reconstruction technique, is derived from compressed sensing $[10,11]$ and has obtained potential applications in several fields, i.e., image processing, collaborative filtering, spectrum sensing. For a low rank matrix[12], MC technology can complement missing data in a observation matrix with a high probability by transforming rank minimum problem into nuclear norm optimization problem[13]. In radar applications, the introduction of $\mathrm{MC}$ can significantly save processing costs and reduce the number of antennas and sampling devices. In Ref.[14,15], MC is applied to MIMO communication system. In Ref.[16], a method of wideband DOA based on co-prime arrays with subNyquist sampling is proposed. In recent years, some scholars have studied the DOA estimation based on coprime arrays. The method in Ref.[17] improves the degree of freedom of DOA estimation. Then in Ref.[18], the proposed method applies MC in coprime arrays. Nowadays, the theory has also made a great deal of achievements in fast algorithm and accurate reconstruction $[19,20]$ and showed great research value and broad application prospect. However, sampling from time domain is indispensable and 2D DOA for URAs cannot be solved by those above methods.
Based on the above analysis, this paper proposes a 2D-DOA estimation method combining Accelerated Proximal Gradient singular value thresholding with Multiple Signal Classification(APG-MUSIC) algorithm under optimized sampling array by introducing $\mathrm{MC}$ into 2D-DOA in planar array. Firstly, a 2D-DOA estimation signal model is built by radiation source data in sparse array, which is proved to meet the properties of low rank and NSP. Then, to improve the performance of received signal matrix reconstruction, we provide a Genetic Algorithm(GA) off-line optimization method which aims at minimizing matrix reconstruction error. Finally, APG and MUSIC are respectively employed to reconstruct received signal matrix and estimate the direction of wave arrived. Compared with conventional 2D-DOA algorithms, the proposed method can make full use of the known array elements and reduce the average sidelobes level.

\section{Signal model of 2D-DOA based on MC}

\subsection{Signal model of 2D-DOA in planar array}

A uniform rectangular array(URA) is shown in figure 1. Assume that the planar array is composed of a total number of $M_{x} \times M_{y}$ elements, and the number of entries in x-direction and y-direction is $M_{x}$ and $M_{y}$ respectively, with the corresponding array spacings $d_{x}$ and $d_{y}$. The elevation and azimuth angles of targets are $\theta$ and $\phi$ respectively.

Suppose that the received signals contain $k$ targets and the number of snapshot is $N . s_{i}(t)(t=1,2, \cdots, N)$ 


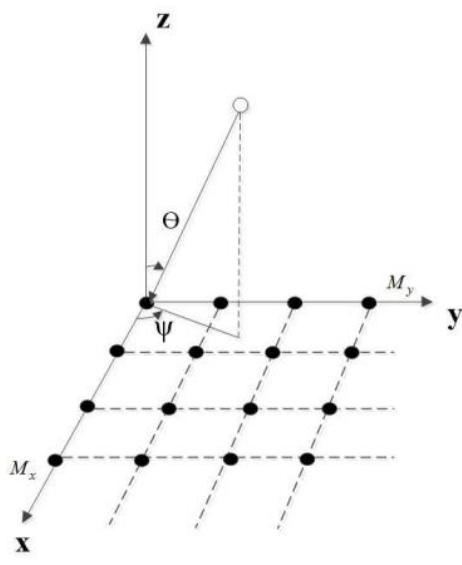

Fig. 1. Structure of uniform rectangular array

represents the waveform of $i$-th $(i=1,2, \cdots, k)$ target, $\left(\theta_{i}, \phi_{i}\right)$ refers to the elevation and azimuth angle of $i$-th target. Considering that the received signals are uncorrelated and narrow-band in far field, thus the received signals of $M_{x}$ elements in x-direction can be written as follows:

$$
\mathbf{X}_{x}(t)=\mathbf{A}_{x} \mathbf{S}(t)+\mathbf{N}_{x}(t)
$$

where $\mathbf{S}(t)=\operatorname{diag}\left(\boldsymbol{s}_{i}(t)\right)$ is a diagonal matrix, $\mathbf{N}_{x}(t)$ is the noise matrix. $\mathbf{A}_{x}$ is the steering vector in $\mathrm{x}$ direction, which can be given by

$$
\mathbf{A}_{x}=\left[\boldsymbol{a}_{x}\left(\theta_{1}, \phi_{1}\right), \boldsymbol{a}_{x}\left(\theta_{2}, \phi_{2}\right), \ldots, \boldsymbol{a}_{x}\left(\theta_{k}, \phi_{k}\right)\right]
$$

Suppose $\lambda$ is the wavelength of received signal, then $\boldsymbol{a}_{x}\left(\theta_{i}, \phi_{i}\right)$ can be represented as a matrix form below:

$$
\boldsymbol{a}_{x}\left(\theta_{i}, \phi_{i}\right)=\left[\begin{array}{c}
1 \\
\exp \left(j 2 \pi d_{x} \sin \theta_{i} \cos \phi_{i} / \lambda\right) \\
\vdots \\
\vdots \\
\exp \left[j 2 \pi\left(M_{x}-1\right) d_{x} \sin \theta_{i} \cos \phi_{i} / \lambda\right]
\end{array}\right]
$$

Similar to above analysis, the received signal model of $M_{y}$ array elements in y-direction can be expressed as:

$$
\mathbf{X}_{y}(t)=\mathbf{A}_{y} \mathbf{S}(t)+\mathbf{N}_{y}(t)
$$

where $\mathbf{A}_{y}$ is the steering vector of $\mathrm{y}$-direction which can be represented as

$$
\mathbf{A}_{y}=\left[\boldsymbol{a}_{y}\left(\theta_{1}, \phi_{1}\right), \boldsymbol{a}_{y}\left(\theta_{2}, \phi_{2}\right), \cdots, \boldsymbol{a}_{y}\left(\theta_{k}, \phi_{k}\right)\right]
$$

where $\boldsymbol{a}_{y}\left(\theta_{i}, \phi_{i}\right)$ is defined as the following vector form:

$$
\boldsymbol{a}_{y}\left(\theta_{i}, \phi_{i}\right)=\left[\begin{array}{c}
1 \\
\exp \left(j 2 \pi d_{y} \sin \theta_{i} \sin \phi_{i} / \lambda\right) \\
\vdots \\
\vdots \\
\exp \left[j 2 \pi\left(M_{y}-1\right) d_{y} \sin \theta_{i} \sin \phi_{i} / \lambda\right]
\end{array}\right]
$$

Above all, the received signal of this planar array can be written as

$$
\mathbf{X}(t)=\mathbf{A}_{x} \mathbf{S}(t) \mathbf{A}_{y}{ }^{T}+\mathbf{N}(t) \quad t=1,2, \cdots, N
$$

In practice, the signal power is usually much higher than the noise power, thus it can be seen that the plane uniform rectangular array has a relatively high received signal-to-noise ratio(SNR) for far-field narrow-band uncorrelated signals. Thus, in the equation (7), $\mathbf{N}(t)$ will have no effect on the rank of $\mathbf{X}(t)$, and $\operatorname{rank}(\mathbf{X}(t)) \leq \operatorname{rank}(\mathbf{S}(t))=k$, that is $\mathbf{X}(t)$ is a lowrank matrix.

\subsection{NSP property of 2D-DOA estimation model}

The received signal in complete array can be recovered from the sparse array signal by MC algorithm. The received signal matrix of full rectangular array is expressed as $\mathbf{X}_{R}(t)$ and the received signal of sparse array can be $\mathbf{X}_{s}(t)$. Let a sampling operator be $P_{\Omega}$, thus 2D-DOA signal model based on MC is

$$
\left\{\begin{array}{cc} 
& \min \left\|\mathbf{X}_{R}(t)\right\|_{*} \\
\text { s.t. } & P_{\Omega}\left(\mathbf{X}_{R}(t)\right)=P_{\Omega}\left(\mathbf{X}_{s}(t)\right)
\end{array}\right.
$$

The null space of orthogonal projection is $\operatorname{Null}\left(P_{\Omega}\right)=\left\{\mathbf{M} \in \mathbf{R}^{n_{1} \times n_{2}}: P(\mathbf{M})=\mathbf{0}\right\}$. According to NULL Space Property, it is difficult to recover the matrix which exists in the null space of orthogonal projection by MC. Let us analyse the signal model established in previous section. Since the vector $\boldsymbol{a}_{x}\left(\theta_{i}, \phi_{i}\right) \neq \mathbf{0}$, any element in the matrix $\mathbf{A}_{x}$ consisting of such vectors is non-zero, too. Likewise, any element in the matrix $\mathbf{A}_{y}$ is also non-zero. And the diagonal elements in the diagonal matrix $\mathbf{S}(t)$ is non-zero. Then we can conclude that elements in $\mathbf{X}_{R}(t)$ are all non-zero. Consequently, arbitrary orthogonal projection meets $P_{\Omega}\left(\mathbf{X}_{R}(t)\right) \neq \mathbf{0}$, that is, $\mathbf{X}_{R}(t)$ meets NSP and can be recovered in higher probability by $\mathrm{MC}$.

\section{GA-based of optimization of sampling elements}

In the signal model based on $\mathrm{MC}$, a small number of elements in planar array need to be selected to construct sparse array. Generally speaking, the array elements can be sampled uniformly at random. In this paper, genetic algorithm(GA) is implemented to optimize the selection of sampling elements, which makes the recovery error of MC smaller. If the number of sampled entries $m$ obeys $m \geq \operatorname{Cr}\left(n_{1}+n_{2}-r\right)$ for some positive constant $C$, the complete received matrix can be recovered with high probability. Let $m \geq 2 r\left(n_{1}+n_{2}-r\right)$, then we convert the problem to minimizing recovery error, which can be represented as:

$$
\min S N E=f\left(\mathrm{~d}_{1}, \mathrm{~d}_{2}, \cdots, \mathrm{d}_{m}\right)
$$

where $\left(\mathrm{d}_{1}, \mathrm{~d}_{2}, \cdots, \mathrm{d}_{m}\right)$ is the position vector set of the array and fit is a fitness function characterizing the reconstruction error of planar array signals, which can be denoted as

$$
\text { fit }\left(\mathrm{d}_{1}, \mathrm{~d}_{2}, \cdots, \mathrm{d}_{m}\right)=S N E=\left\|\mathbf{X}_{\text {full }}-\mathbf{X}_{M C}\right\|_{2} /\left\|\mathbf{X}_{\text {full }}\right\|_{2}
$$


where $\mathbf{X}_{\text {ful }}$ is the complete received signal matrix and $\mathbf{X}_{M C}$ is the recovered matrix. The flow chart of optimizing the sampling elements by GA is as shown in figure 2 .

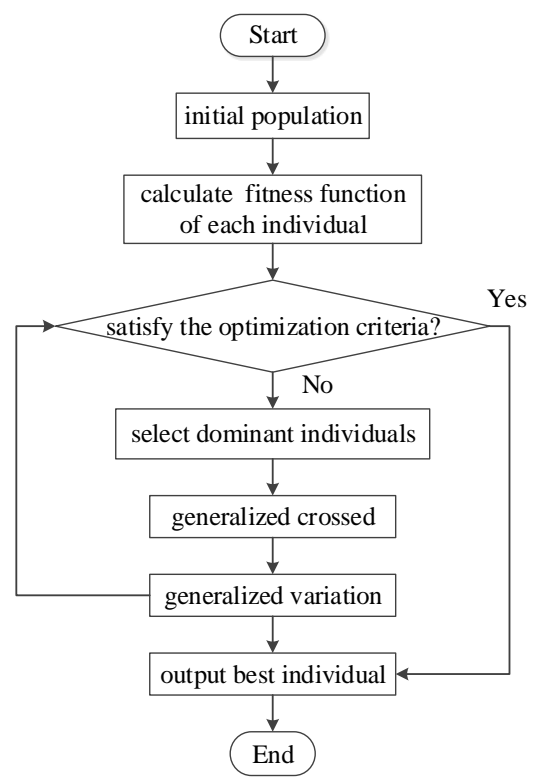

Fig. 2. The flow chart of optimizing the selection by GA

\section{APG-MUSIC algorthim}

We proposes a APG-MUSIC algorithm to solve the contradiction between the number of array elements and the average sidelobes level. Firstly, the complete received signal matrix $\mathbf{X}_{R}(t)$ can be achieved by APG algorithm from $\mathbf{X}_{s}(t)$, then we vectorize the matrix and solve its auto-correlation matrix. Finally, the targeted angles can be estimated from the spatial spectrum by MUSIC.

The established sparse signals model baesd on MC can be transformed into APG problem as follows:

$$
\min \mu\left\|\mathbf{X}_{R}(t)\right\|_{*}+\frac{1}{2}\left\|P_{\Omega}\left(\mathbf{X}_{R}(t)\right)-\mathbf{X}_{s}(t)\right\|_{F}^{2}
$$

The problem mentioned above can be solved by corresponding iterations and each iteration uses formula (12) to update the current matrix:

$$
S_{\tau}(\mathbf{G})=\mathbf{U} \operatorname{Diag}\left((\sigma-\mu / \tau)_{+}\right) \mathbf{V}^{T}
$$

where ${ }^{\mu}$ is a positive constant and $\mathbf{U} / \mathbf{V} / \sigma$ are obtained from the singular value decomposition(SVD). Given

$$
\mu>0
$$

let $\mathbf{X}^{0}=\mathbf{X}^{-1}=\mathbf{X}_{s}(t), t^{0}=t^{-1}=1, k=0,1,2, \cdots, \quad$ the iteration process from $\mathbf{X}^{k}$ to $\mathbf{X}^{k+1}$ can be given by the following steps:

1. Set $\mathbf{P}^{k}=\mathbf{X}^{k}+\frac{t^{k-1}-1}{t^{k}}\left(\mathbf{X}^{k}-\mathbf{X}^{k-1}\right)$;

2. $\mathbf{G}^{k}=\mathbf{P}^{k}-\left(\tau^{k}\right)^{-1} P_{\Omega}{ }^{*}\left(P_{\Omega}\left(\mathbf{P}^{k}\right)-\mathbf{X}_{s}(t)\right)$, where $\tau_{k}>0$;

3. Obtain $\mathbf{U}, \mathbf{V}, \sigma$ from the SVD of $\mathbf{G}^{k}$ then do contraction operator on it, that is,

$\mathbf{X}^{k+1}=S_{\tau^{k}}\left(\mathbf{G}^{k}\right)=\mathbf{U} \operatorname{Diag}\left(\left(\sigma-\mu / \tau^{k}\right)_{+}\right) \mathbf{V}^{T}$
4. Update $t^{k+1}=\frac{1+\sqrt{1+4\left(t^{k}\right)^{2}}}{2}$;

5. If the stop condition of iterations is satisfied, then end the loops and the output is turned out to be $\mathbf{X}^{\text {opt }}$, that is, $\mathbf{X}^{\text {opt }}=\mathbf{X}^{k}$.Otherwise, go to step 1 .

The stop condition can be chosen as shown in equation (13).

$$
\frac{\left\|\mathbf{X}^{k}-\mathbf{X}^{k-1}\right\|_{F}}{\max \left\{\left\|\mathbf{X}^{k}\right\|_{F}, 1\right\}}<\varepsilon
$$

where $\varepsilon$ is a tolerance for stopping criterion. The contraction operator in Step3 can be denoted as

$$
S_{a}(x)=\left\{\begin{array}{cc}
x-a & x>a \\
x+a, & x<-a \\
0, & \text { others }
\end{array}\right.
$$

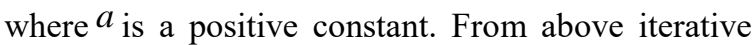
process, regardless of the noise, the structure of the received signal can be represented as

$$
\hat{\mathbf{X}}_{R}(t)=\mathbf{A}_{x} \mathbf{S}(t) \mathbf{A}_{y}^{*}=\mathbf{U} \boldsymbol{\Sigma} \mathbf{V}^{*}
$$

Vectorize $\hat{\mathbf{X}}_{R}(t)$, we can obtain the vector form $\boldsymbol{x}_{R}(t)$.

$$
\boldsymbol{x}_{R}(t)=\sum_{i=1}^{k} \boldsymbol{a}\left(\phi_{i}, \theta_{i}\right) \boldsymbol{s}_{i}(t)+\boldsymbol{n}(t) \quad t=1,2, \cdots, N
$$

Compute the auto-correlation matrix $\mathbf{R}_{x}$ of the received signal vector $\mathbf{R}_{x}$ :

$$
\mathbf{R}_{x}=\frac{1}{N} \sum_{n=1}^{N} \boldsymbol{x}(n) \boldsymbol{x}^{H}(n)
$$

where $N$ is the number of snapshots.

Then solve the eigen-decomposition of the matrix $\mathbf{R}_{x}$, we can get

$$
\mathbf{R}_{x}=\mathbf{U}_{S} \boldsymbol{\Sigma}_{S} \mathbf{U}_{S}{ }^{H}+\mathbf{U}_{N} \boldsymbol{\Sigma}_{N} \mathbf{U}_{N}{ }^{H}
$$

where $\mathbf{U}_{S}$ is the signal subspace consisting of eigenvectors of ${ }^{k}$ larger eigenvalues and $\mathbf{U}_{\mathrm{N}}$ is the noise subspace composed of the remaining eigenvectors.

The signal and noise subspace also satisfies:

$$
\mathbf{U}_{S} \mathbf{U}_{S}^{H}+\mathbf{U}_{N} \mathbf{U}_{N}^{H}=\mathbf{I}
$$

Therefore the $2 \mathrm{D}$ spatial spectrum can be obtained to conduct spectral peak search:

$$
P_{2 D-M U S I C}=\frac{1}{\boldsymbol{a}^{H}(\theta, \phi) \mathbf{U}_{N} \mathbf{U}_{N}^{H} \boldsymbol{a}(\theta, \phi)}
$$

where $\boldsymbol{a}(\theta, \phi)=\boldsymbol{a}_{y}(\theta, \phi) \otimes \boldsymbol{a}_{x}(\theta, \phi)$.

\section{Simulations and analysis}

In this section, computer simulations are carried out to demonstrate the effectiveness and superiority of the proposed algorithm. Assume that the URA contains $64 \times 64=4096$ entries totally and elements spanned spacing in $\mathrm{x}$-direction and $\mathrm{y}$-direction equals to half of the wavelength, that is, let $d_{x}=d_{y}=\lambda / 2$.GA algorithm 
is employed to optimize the sampling array. We choose $\mathrm{m}=1800$. Consider three incoherent sources in the space. Their elevation angle $\theta$ and azimuth angle $\phi$ are $\left(10^{\circ}, 15^{\circ}\right),(30,25)$ and $\left(50^{\circ}, 35^{\circ}\right)$ respectively.

Experiment1 2D-DOA estimation in sparse array is discussed. Set Signal Noise Ratio(SNR) as $10 \mathrm{~dB}$ and the number of snapshots as 150 . The 2D spatial spectrum of randomly sampling array by MUSIC algorithm is shown in figure 3. As shown in the pattern, the average sidelobes level is $-2.99 \mathrm{~dB}$. It can be concluded that due to the sparsity of the array, the average sidelobes level rises and the spectrum broadens, which will seriously affects the accuracy of 2D-DOA estimation.

Experiment2 2D-DOA estimation based on APGMUSIC with optimized sampling array is discussed. We utilize optimized sparse array to reconstruct received signal matrix by APG and then implent MUSIC to estimate the spatial spectrum. The result is shown in figure 4 . It can be easily seen that the average sidelobes level is $-56.31 \mathrm{~dB}$. It is easy to find that by comparison with figure 3 the proposed method has better performance in 2D-DOA estimation under the same sampling size. It is mainly due to its ability to reconstruct the received signal matrix with a higher probability, which increases accumulated SNR to some extent. Therefore, the APG-MUSIC 2D-DOA estimation method can effectively suppress sidelobes level.

Experiment3 The relationship between sampling array selection and reconstruction performance is verified. We compare uniformly random sampling with GA optimized selection when the sampling number is $1500,1800,2100,2400$ and 2700 respectively. It can be easily seen from figure 5 that GA optimized sampling has better performance in matrix reconstruction and significantly reduces recover error. The experimental results show the importance and research value of sampling array optimization in array signal reconstruction.

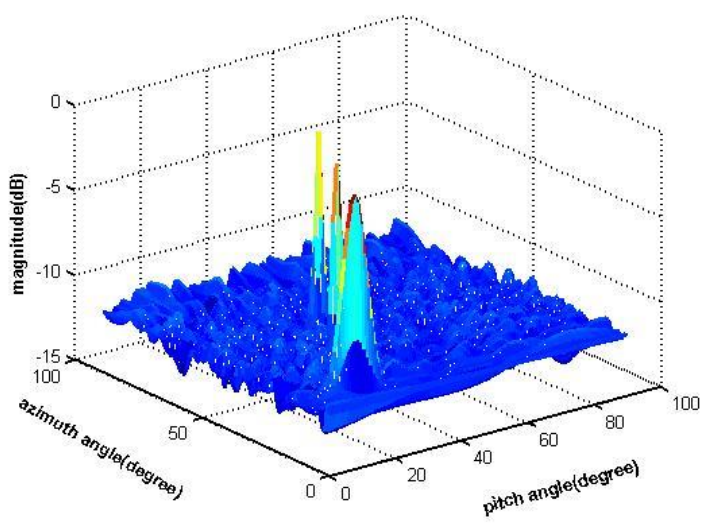

Fig.3. 2D-DOA estimation by MUSIC under randomly sampling array

\section{Conclusion}

An APG-MUSIC 2D-DOA estimation method based on sparse sampling array optimized is proposed in this paper. Compared with conventional 2D-DOA estimation methods, the proposed method not only achieves low sidelobes level, but also reduces the sampling number dramatically and improves the utilization rate of array, which has high research value and broad application prospect. In my paper, the noise is not taken into consideration. However, how to recover a complete matrix accurately in a noisy environment is an important research direction. Consequently, the related problems of 2D-DOA estimation based on MC under noise need to be further studied.

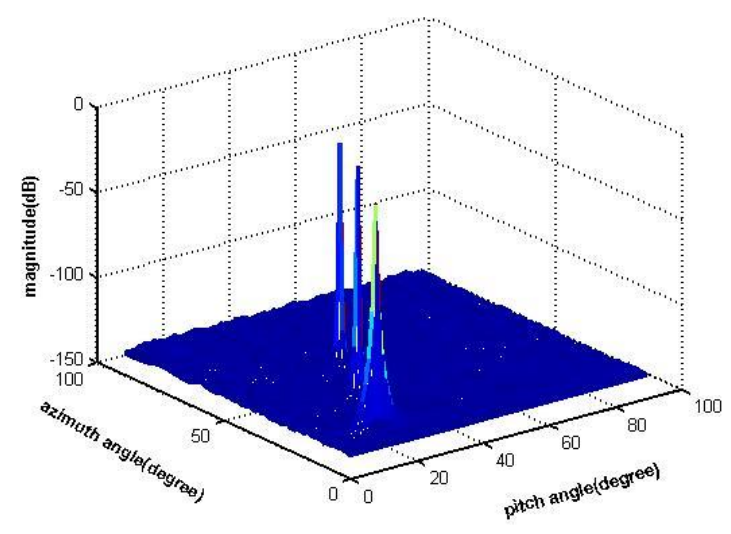

Fig. 4. 2D-DOA estimation by APG-MUSIC under GA optimized selection

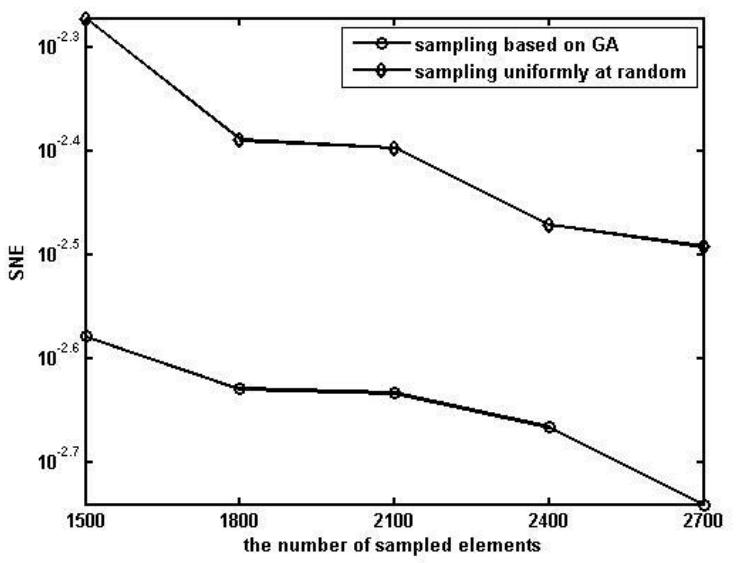

Fig. 5. SNE verus the sampling number in two different methods

\section{References}

1. DUDGEON D E. Fundamentals of digital array processing $[\mathrm{J}]$. Proceedings of the IEEE,1977,65(6):898-904.

2. Zhang Xiaofei. Theory and application of array signalprocessing [M]. Beijing:National Defence Industry Press,2010.

3. Luo Zheng,Zhang Min,Li Pengfei. The new algorithm of two-dimensional DOA based on autocorrelation matrix with high power[J]. Journal of aviation 2012,33(4):696-704. 
4. Pal P, Vaidyanathan P P.A grid-less approach to underdetermined direction of arrival estimation via low rank matrix denoising $[\mathrm{J}]$. Signal Processing Letters, IEEE,2014,21(6): 737-741.

5. Cands E J,Plan Y.Matrix Completion WithNoise[J]. Proceedings of the IEEE,2009.

6. Cands EJ, Eldar YC, Strohmer T, Voroninski V. Phase retrieval via matrix completion. SIAM J Imaging Sci 2011;6(1):199225.

7. Cands EJ, Emmanuel S, Recht B. Exact matrix completion via convex optimization. Commun ACM 2012;55(6):1119.

8. Chen C, He B, Yuan X. Matrix completion via an alternating direction method.IMA J Numer Anal 2012;32(1):22745.

9. Toh Kim-Chuan, Yun Sangwoon. An accelerated proximal gradient algorithm for nuclear norm regularized linear least squares problems. Pacific J Optim 2010;6(3):61540.

10. Donoho DL.Compressed sensing. IEEE Trans Inform Theory 2006;52(4):1289306.

11. Duarte MF, Baraniuk RG. Spectral compressive sensing. Appl Comput Harmon Anal 2013;35(1):11129.

12. Yuan X, Yang J.Sparse and low-rank matrix decomposition via alternating direction methods[J]. preprint,2009,12.

13. Recht B, Xu W, Hassibi B. Necessary and sufficient conditions for success of the nuclear norm heuristic for rank minimization.Eprint Arxiv 2008;16(5):306570.

14. Li B, Petropulu A. Spectrum sharing between matrix completion based MIMO radars and a MIMO communication system[C]. IEEE International Conference on Acoustics, Speech and Signal Processing. IEEE,2015:2444-2448.

15. Zhang N, Xie C L, Tang J, et al. Three-dimensional extensions of matrix completion based MIMO radar[C]//Iet International Radar Conference. 2015:4. -4.

16. Wanghan L V, Wang H, Liu F, et al. Wideband DOA Estimation Based on Co-Prime Arrays with Sub-Nyquist Sampling[J]. Ieice Transactions on Fundamentals of Electronics Communications and Computer Sciences,2016, E99.A(9):1717-1720.

17. Chen Chuan, Guo Yong, Jia Yong. A DOA estimation method based on dual frequency coprime array[J]. Computer Application Research, 2017,34(05):1459-1462.

18. Wu Chenxi, Zhang Min, Wang Ke. A underdetermined DOA estimation method of coprime array based on matrix completion[J]. Engineering Science and Technology,2017,49 (05): 156-163.

19. Hu Y, Zhang D, Ye J, et al. Fast and accurate matrix completion via truncated nuclear norm regularization. IEEE Trans Pattern Anal Mach Intell 2013;35(9):211730.

20. Recht B. A simpler approach to matrix completion. J Mach Learn Res 2011;12(4):3413-30. 